SU-ITP-92/14

SSCL-Preprint-116 April 1992

(T/As)

\section{Potential Motion for Thomas-Fermi Non-Topological Solitons}

\author{
SAFI BAHCALL \\ Department of Physics, Stanford University, Stanford, California 94305
}

and

BRYAN W. LYNN ${ }^{\dagger}$

Department of Physics, Stanford University, Stanford, California 94305

and

Superconducting Super Collider Laboratory, Dallas, Texas, 75237-3946

\section{ABSTRACT}

In the Thomas-Fermi approximation to theories of coupled fermions and scalars, the equations for spherically-symmetric non-topological solitons have the form of potential motion. This gives a straightforward method for proving the existence of non-topological solitons in a given theory and for finding the constant-density, saturating solutions.

* Work supported in part by an NSF Graduate Fellowship and NSF contract NSF-PHY-8917438.

$\dagger$ Work supported in part by the NSF, contract NSF-PHY-8917438, and by the Universities Research Association Inc. with Department of Energy contract DE-AC35-89ER40486.

\section{DISTRIBUTION OF THIS DOCUMENT IS UNLIMITEO DO}

Solitons in field theory, in the sense usually used in the literature, are finiteenergy, non-singular, localized classical solutions to field equations. These solutions can be stable either because of topological reasons, with boundary conditions at infinity preventing decay into lower energy states, or because the solutions themselves are the lowest energy states. We will consider the second kind here, which have been called 'non-topological solitons.'.1.2]

In purely scalar field theories, time-dependent non-topological soliton solutions have been explicitly constructed. ${ }^{[2.3 .4]}$ Such solutions correspond in a semiclassical approximation to coherent states of the full quantum theory. In fermion theories, the situation is more complicated: occupation numbers never get larger than one, so classical solutions are never a good approximation. The Dirac equation must be solved to find the true quantum states which are then filled to form a Fermi sea. This has been done for systems with small numbers of fermions, with a boson interaction added as a background field in the Dirac equation, and applied as a model of hadrons. ${ }^{|\theta|}$ For systems with many fermions, however, it is impossible to solve for all the quantum states. Instead, there is an approximation, due to Thomas and Fermi, ${ }^{[7]}$ that can be used when solutions have fields which are sufficiently slowly-varying functions of position: the fermions may be treated as free particles stacked in a Fermi sea with a position-dependent Fermi momentum. We are interested in theories with fermions coupled to scalars, which provide an attractive force to overcome Fermi repulsion and allow bound states. The Thomas-Fermi approximation in this case incorporates the effects of fermions into the scalar field equation of motion through the assumption of a filled Fermi sea with a Fermi momentum that depends on the value of that field. It was first introduced in the context of non-topological solitons in Ref. 1, followed by many papers applying this idea to specific models, particularly to nuclei (see Ref. 5 for a review).

Here we show that in the Thomas-Fermi approximation, the three dimensional spherically-symmetric scalar field equation has the form of potential motion. This follows simply from energy-momentum conservation, with the potential being the total pressure in the problem, and therefore generalizes easily. As 
for scalar field theories, where the mechanical analogy is often used, the potential motion form for fermion field theories is useful for determining the existence of solitons in a given theory and showing whether there are special solutions which can be any size and have constant energy and number densities. Because of the resemblance to what is called the saturation of the interiors of heavy nuclei, we call the special solutions saturating.

Consider for the moment just one scalar field $\phi$ and one fermion field $\psi$. Write the Lagrangian as

$$
L=L_{\phi}+L_{\psi}
$$

where $L_{\phi}$ contains those terms that involve only $\phi$,

$$
L_{\phi}=\frac{1}{2}\left(\partial_{\mu} \phi\right)^{2}-U(\phi)
$$

and $L_{\psi}$ contains the rest, including interactions between $\phi$ and $\psi$ :

$$
L_{\psi}=\bar{\psi}(i \not \partial-m(\phi)) \psi+\ldots
$$

The Thomas-Fermi approximation consists of neglecting terms involving spatial derivatives of $\phi$ in the square of the Dirac equation that follows from (3), based on the assumption that $\phi$ is slowly varying. Then the fermion states are those of free particles with a dispersion relation which depends on on $\phi(\vec{x})$. Derivative interactions in eqn. (3) contribute at the same order as terms that are being neglected, and therefore have no effect within the approximation.

We can write the conservation of energy-momentum in this theory as

$$
\partial_{\mu}\left(T_{\phi}^{\mu \nu}+T_{\psi}^{\mu \nu}\right)=0
$$

with

$$
T_{\phi}^{\mu \nu}=\frac{\partial L_{\phi}}{\partial\left(\partial_{\mu} \phi\right)} \partial^{\nu} \phi-g^{\mu \nu} L_{\phi}
$$

and the energy-momentum tensor for the fermions given by the perfect flaid of the Thomas-Fermi approximation,

$$
T_{\psi}^{\mu \nu}=\left(E_{\psi}+P_{\psi}\right) U^{\mu} U^{\nu}-P_{\psi} g^{\mu \nu}
$$

In the rest frame of the fluid the velocity four-vector $U^{\mu}=\delta_{0}^{\mu}$. Since we are neglecting the dependence of $P_{\psi}$ on derivatives of $\phi$, eqn. (4) becomes

$$
\partial_{\nu} \phi\left(\partial_{\mu} \frac{\partial L_{\phi}}{\partial\left(\partial_{\mu} \phi\right)}-\frac{\partial L_{\phi}}{\partial \phi}-\frac{\partial P_{\psi}}{\partial \phi}\right)=-\delta_{\nu, 0}\left(\dot{E}_{\psi}+\dot{P}_{\psi}\right)
$$

For static fermion densities, this gives

$$
\partial_{\mu} \frac{\partial L_{\phi}}{\partial\left(\partial_{\mu} \phi\right)}-\frac{\partial L_{\phi}}{\partial \phi}=\frac{\partial P_{\psi}}{\partial \phi}
$$

The right hand side describes the effect of the fermion fluid on the scalar field equation of motion.

For the Lagrangian (2), static, spherically-symmetric solutions in three space dimensions satisfy

$$
\phi^{\prime \prime}+\frac{2}{r} \phi^{\prime}=-\frac{\partial}{\partial \phi}\left(P_{\psi}-U\right)
$$

where the prime denotes differentiation with respect to the radial coordinate $r$. This equation has the form of potential motion for a particle at position $\phi$ and time $r$ moving in the potential $V=P_{\psi}-U$, with a friction term whose coefficient is inversely proportional to time.

This form of potential motion can be generalized to more complicated theories. For a theory with $n$ scalars and any number of fermions, $\vec{\phi}$ rolls as a particle in an $n$-dimensional space moving in a potential $P_{\psi}-U(\vec{\phi})$, where $P_{\psi}$ is the sum of all the Fermi pressures. In theories with vector fields, the transverse component will satisfy the same rolling equation, but the potential has the opposite sign, $V=U-P_{\psi}$, so that the rolling does not apply to theories 
containing both scalars and vectors. For a theory with a charged scalar field, scalar field solutions will have a time dependence which adds a quadratic term to the potential $U$ (see Ref. 4). Gravitational effects, which become relevant when the solution becomes heavy enough, can also be included. ${ }^{[8,9]}$ Theories in which the scalar Lagrangian has a more complicated kinetic term, such as chiral perturbation theory, can often be transformed into the form (2), so the equations for the transformed field will have the form of potential motion. For simplicity, we consider here only the one fermion, one scalar theory given by eqns. (1)-(3).

With a given Fermi energy and appropriate boundary conditions on $\phi$, $\phi(r \rightarrow \infty)=\phi_{0}$ (vacuum value) and $\phi^{\prime}(0)=0$, eqn. (9) can be solved for the field configuration $\phi(r)$, which is then inserted into the dispersion relation derived from eqn. (3) to give the Fermi momentum $k_{F}(r)$. The number density, energy density, and pressure of the fermions are given by the usual expressions involving $k_{F}(r)$. $^{(0)}$ The form of eqn. (9) allows the spherically-symmetric saturating solutions to be found without solving any differential equations.

Saturating solutions have constant values for the fields out to large distances, which means that $\phi$ sits still for a long time. In this case the friction term in eqn. (9) can be ignored, because $r$ is large before $\phi$ starts moving. Saturating solutions therefore occur when $\phi$ rolls between two degenerate hills. ${ }^{\star}$ One hill occurs at the vacuum value of $\phi$, call it $\phi_{0}$, which is the value to which $\phi$ must roll at large times to insure the energy is finite. The other hill, from which $\phi$ begins to roll at $r=0$, is found by solving the algebraic equations

$$
\begin{aligned}
P_{\psi}-U & =0 \\
\frac{\partial}{\partial \phi}\left(P_{\psi}-U\right) & =0
\end{aligned}
$$

for $\epsilon_{F}$, the Fermi energy, and $\phi_{i n}$, the value of the scalar field in the interior of the saturating solution. The solution exists, that is, there is a surface connecting

$\star$ For a detailed discussion of this in a charged boson theory see, for example, ref. 4. In that theory $P_{\psi}$ is replaced by $\frac{1}{2} \mu|\phi|^{2}$, coming from a time dependence in the phase of $\phi$. Otherwise, the story about rolling around and saturating solutions is just the same. the interior and exterior, provided $P_{\psi}-U<0$ for $\phi_{i n}<\phi<\phi_{0}$. In practice, these solutions can be found by graphing $U(\phi)$ and $P_{\psi}\left(\epsilon_{F}, \phi\right)$ then adjusting $\epsilon_{F}$ until the two curves are tangent at a point other than the vacuum.

The properties of the saturating solutions follow as before from knowing $\epsilon_{F}$ and $\phi$, where $\phi=\phi_{i n}$ is now constant. In particular, the volume energy density is given by $U\left(\phi_{i n}\right)+E_{\psi}\left(\epsilon_{F}, \phi_{i n}\right)$ and can be used to determine whether the solution is energetically bound. Because the surface energy is smaller than the volume energy by a factor $\sim 1 / R$, the volume energy is a good approximation to the total energy when the radius $R$ is large. We note that the Thomas-Fermi approximation is extremely good in the interior of these solutions, where the field derivatives are very small, but is less good in the surfaces. Further discussion of these solutions and their applications can be found in previous papers. ${ }^{[0,10]}$

In this paper we have shown that in theories of coupled fermions and scalars, the spherically-symmetric scalar field equation will have the form of potential motion when the fermions are treated in the Thomas-Fermi approximation. In this case it is straightforward to check whether the field theory has non-topological soliton solutions, either saturating or non-saturating. The most interesting application we know of for these results is in nuclear plysics: it is possible that nuclei might be described as solitons in either an effective Lagrangian cooked up for such a purpose, such as the Walecka model ${ }^{[5.12]}$ or, even more interesting, in effective chiral Lagrangians used to describe hadronic scattering. ${ }^{[12]}$ 


\section{REFERENCES}

1. T.D. Lee and G.C. Wick, Phys. Rev. D 9 (1974) 2291.

2. R. Friedberg, T.D. Lee, and A. Sirlin, Phys. Rev. D 13, 2739 (1976); Nucl. Phys. B115, 1 (1976); B115, 32 (1976).

3. G. Rosen, J. Math. Phys. 9 (1968) 996.

4. S. Coleman, Nucl. Phys. B 262 (1985) 263.

5. B.D. Serot and J.D. Walecka in Advances in Nuclear Physics, vol. 16, ed. J.W. Negel and E. Vogt (Plenum, New York, 1985) p.1.

6. R. Friedberg and T.D. Lee, Phys. Rev. D 15, 1694 (1977); 16, 1096 (1977); 18, 2623 (1978); T.D. Lee, Particle Physics and Introduction to Field Theory (Harwood Academic, New York, 1981).

7. L.H. Thomas, Proc. Camb. Phil. Soc. 23 (1927) 542.

E. Fermi, Zeits. F. Physik 48 (1928) 73.

8. T.D. Lee and Y. Pang, Plys. Rev. D 35 (1987) 3678.

9. S. Balicall, B.W. Lynn, and S.B. Selipsky, Nucl. Phys. B 325 (1989) 606 .

10. S. Bahcall, B.W. Lynn, and S.B. Selipsky, Nucl. Phys. B 331 (1990) 67.

11. J.D. Walecka, Annals Phys. 83 (1974) 491.

12. B.W. Lynn, "A Theory of Nuclei from the Effective Chiral Lagrangian", SU-ITP-92/3.

\section{DISCLAIMER}

This report was prepared as an account of work sponsored by an agency of the United States Government. Neither the United States Government nor any agency thereof, nor any of their employees, makes any warranty, express or implied, or assumes any legal liability or responsibility for the accuracy, completeness, or usefulness of any information, apparatus, product, or process disclosed, or represents that its use would not infringe privately owned rights. Reference herein to any specific commercial product, process, or service by trade name, trademark, manufacturer, or otherwise does not necessarily constitute or imply its endorsement, recommendation, or favoring by the United States Government or any agency thereof. The views and opinions of authors expressed herein do not necessarily state or reflect those of the United States Government or any agency thereof. 


\section{DISCLAIMER}

Portions of this document may be illegible in electronic image products. Images are produced from the best available original document. 